\title{
CARTA A LOS ROMANOS DE KARL BARTH: SUS ANTECEDENTES EN EL PENSAMIENTO DE KIERKEGAARD Y SU INFLUENCIA EN LA FILOSOFÍA EXISTENCIAL DEL SIGLO XX
}

\author{
Marcio Gimenes de Paula \\ Universidad de Brasília, Brasil
}

\section{Resumen}

El objetivo de este artículo es investigar cómo la Carta a los romanos, obra clásica del teólogo suizo Karl Barth, posee una fuerte influencia de la interpretación de Kierkegaard sobre el cristianismo y cómo tal obra, a su vez, influyó en toda una generación de pensadores de la filosofía existencialista en el siglo XX. Así, la propuesta está dividida en dos partes centrales: la primera pretende investigar la influencia de Kierkegaard en Karl Barth y la segunda desea observar cómo tal obra teológica se torna rica para el existencialismo cristiano -pero no sólo de este- ya en el siglo XX.

Palabras clave: Existencialismo, filosofía de la religión, Kierkegaard, Karl Barth, siglo XX.

\section{Abstract}

The purpose of this article is to investigate how The Letter to the Romans, the classic work of the Swiss theologian Karl Barth, has a strong influence on Kierkegaard's interpretation of Christianity, and how this work in turn influenced a whole generation of thinkers of the existentialist philosophy in the twentieth century. Thus, the proposal is divided into two central parts: the first aims to investigate Kierkegaard's influence on Karl Barth and the second wishes to observe how such a theological work becomes rich for Christian existentialism - but not only for it - in the twentieth century.

Key words: Existentialism, philosophy of religion, Kierkegaard, Karl Barth, 20th century.

Recibido: 7 de agosto de 2019; Aceptado: 20 de septiembre de 2019.

Traducción al español de Yamicela Torres. 


\section{Introducción}

La Carta a los romanos, epístola atribuida al apóstol Pablo es, tal vez, una de esas fuentes inspiradoras de gran reflexión en los ciernes del cristianismo e incluso va más allá de sus fronteras, alcanzando un debate que posteriormente les interesa a las esferas de la ética y la política. Desde los orígenes del cristianismo la epístola encontró acogida en la obra de los grandes teólogos de la Iglesia de todos los tiempos y, de ese modo, Leenhardt parece tener razón en su comentario acerca del grado de alcance de tal texto paulino:

Imposible e inútil sería dar una exhaustiva bibliografía de trabajos consagrados a la Epístola a los romanos o que a esta se refieren en proporción dominante. Informaciones más completas se encontrarán en las obras cuya consulta es, de cualquier forma, indispensable para trabajos más profundos... El siglo XVI vio surgir dos obras capitales, que han ejercido en la historia de la Iglesia la función primordial que a ellas se les reconoce: el Comentario de romanos de Lutero (1515-1516) y los Loci Theologici de Melanchthon (1521). Calvino, por su parte, comentó la Epístola a los romanos con los demás libros del Nuevo Testamento... ${ }^{1}$.

Cabe incluso recordar que la Biblia de Lutero obviamente contiene la traducción de la propia epístola y esa habría sido distintiva en su discusión acerca de la libertad del cristiano y de sus decisiones, tema tan central para el pensamiento luterano. Así, David y Pat Alexander están en lo correcto al percibir el grado de importancia de la epístola a los romanos en el contexto cristiano:

El influjo ejercido por esa epístola ha sido enorme. Esta influyó a grandes hombres -San Agustín, Lutero, Karl Barth...- y a través de ellos ha modelado la historia de la Iglesia, así como ha influido en la vida de innumerables individuos anónimos, hombres y mujeres simples que, al leerla, creyeron en su mensaje y actuaron en consecuencia ${ }^{2}$

La epístola a los romanos data del 57 d.C. En este período, Pablo, a pesar de ser ciudadano romano, todavía no había ido a la capital imperial y, en verdad, sólo se adentrará en ella algunos años después, en la condición de prisionero. La esencia de la epístola reside en el debate central, tan propio del

${ }^{1}$ F.J. Leenhhardt, Epístola aos Romanos- comentário exegético, trad. de Waldyr Carvalho Luz, São Paulo: Aste, 1969, p. 27.

${ }^{2}$ Alexander, David e Pat, O mundo da Bíblia, trad. de Lailah de Noronha, São Paulo: Paulinas, 1985, p. 582. 
cristianismo de los primeros siglos, entre la nueva fe (todavía en formación y muy diferente a como la conocemos hoy) y el paganismo.

La temática de la epístola es bastante significativa y por eso se tornó esencial para las reflexiones teológicas de la fe naciente. Aquí Pablo de posiciona, de un modo singular, entre judíos legalistas, cristianos de diversa procedencia y paganos, en defensa de la fe cristiana y de un tema que será bastante importante para la tradición luterana, a saber, el contexto de la subjetividad y el tema de la libertad humana que, como se podrá observar en el futuro, serán fundamentales para la modernidad.

La carta paulina posee una estructura de 16 capítulos y aquí no nos correspondería enumerarlos o analizarlos con los detalles exegéticos apropiados, pero podemos destacar algunos aspectos importantes. La afirmación de la fe del cristiano, por ejemplo, salta a la vista en el primer capítulo (Rom 1:17) y allí Pablo textualmente afirma que "aquel que es justo vivirá por la fe”. Como sabemos por la tradición, tal texto tendrá una profunda resonancia en Lutero que, al leerlo, se da cuenta de la ineficacia tanto del magisterio eclesiástico como de la práctica de las buenas obras para la salvación de los hombres y, en ese sentido, se convence de que la salvación humana es dada por Dios mediante la fe que, bajo ningún supuesto, se constituye en mérito o en algo que el hombre pueda alcanzar por sus esfuerzos.

En este sentido, la epístola menciona la importante herencia de Abraham (Rom 4) para la fe naciente, y aquí hay una curiosidad. El patriarca de Israel sale ahora de la simple posición de padre de los judíos y alcanza a todos los integrantes de la nueva fe. Es decir, Pablo universaliza su figura, dándole más que la ya conocida posición de patriarca de la fe, el título de nuestro antecesor en ella. Nótese que, en un contexto de cristianismo naciente, donde una parcela del mismo era oriunda del judaísmo y otra compuesta de no judíos que, en general, eran discriminados y tomados como una especie de "discípulos de segunda mano", el texto paulino se muestra vigoroso, valiente e innovador.

El mismo tema de articulación entre la antigua fe judía y la nueva fe cristiana puede ser igualmente observado en el capítulo 5, donde ocurre una aproximación entre Adán y Cristo. Pablo discute la antigua vida de un cristiano y su vida nueva en Cristo. Aquí hay una analogía a la nueva humanidad que ahora posee su naturaleza transformada, tema cristiano por excelencia. Por fin, la epístola abordará el tema de la elección divina, la libertad y la responsabilidad del cristiano ante sus decisiones. De ese modo, 
tal como la definición de fe al comienzo de la epístola, será igualmente provocativa la definición de pecado dada por el apóstol: "Pues todo lo que no proviene de fe es pecado" (Rom 14:23). Ahora bien, lo que Pablo parece intentar realizar aquí es, de hecho, construir un discurso teológico racional y aplicado al cristianismo. Su oposición al paganismo posee, sin dudas, un aspecto de condenación moral a este, pero va más allá en la medida en que tal paganismo parece ser rechazado, ya que no consigue operar racionalmente y por eso, como sospecha Jaeger ${ }^{3}$, parece haber en el cristianismo naciente una nueva paideia, que constituirá el Occidente cristiano y, al mismo tiempo, se diferenciará de la antigua paideia griega y romana, aunque se haya nutrido de algunos de sus aspectos.

Pasemos, entonces, a una evaluación de cómo el pensador danés, Kierkegaard, influyó decisivamente la lectura del teólogo protestante suizo Karl Barth en su clásica interpretación de la Carta a los romanos. El tema de la libertad, de la elección, de la posibilidad de la fe, así como el tema de lo que significa ser apóstol o seguidor del cristianismo es claramente percibido en las obras de Kierkegaard y, obviamente, los toma de su tradición cristiana y protestante, tal como podremos verificar.

I. La influencia de Kierkegaard en Karl Barth: el caso de la "Carta a los
romanos"

La clásica Carta a los romanos de Karl Barth fue publicada en 1918, tal como prueba su primer prefacio. No obstante, en general, es reconocida por ser fechada en 1922, momento de su segunda versión. Fueron escritos seis prefacios para la obra, respectivamente en 1918, 1921, 1922, 1924, 1926, 1928.

El texto de Barth sigue básicamente la misma estructura paulina, o sea, prácticamente cada capítulo del teólogo corresponde a un capítulo del apóstol en la propia epístola. Los temas retomados e interpretados por el teólogo suizo son los mismos temas paulinos, a saber, la justicia humana, la justicia de Dios, la fe, la gracia, la libertad, el espíritu y la Iglesia. Cornelis van der Kooi y Katja Tolstaja, actuales editores de la versión más actualizada de Carta a los romanos de Barth, apuntan en su prefacio un aspecto singular

${ }^{3}$ W. Jaeger, Cristianismo primitivo e paideia grega, trad. de Louro Perez, Lisboa: Edições 70, 2002. 
de la construcción teológica del pensador suizo, a saber, la confrontación entre cristianismo y cultura:

Mientras que en el protestantismo cultural del siglo XIX el cristianismo y la cultura occidental aún eran largamente identificados entre sí y los valores cristianos valían como base y fermento de la sociedad, Barth diferencia ahora entre la revelación y el cristianismo. El cristianismo es un fenómeno cultural, una suma de convicciones y hábitos que dan forma a la existencia humana y estructura a la vida cotidiana. La revelación de Dios, a su vez, debe ser diferenciada de esas expresiones culturales. La fe trata de la confrontación con Dios como categoría de un orden totalmente diferente. Dios es tematizado ahora de forma expresionista y rigurosamente paradójico como algo insólitamente incomprensible y desconocido, que se aproxima peligrosamente ${ }^{4}$.

Aquí podemos percibir cuánto Barth está bajo la influencia kierkegaardiana. El enfrentamiento entre cristianismo y cultura será algo constante en todas las obras del autor danés y puede ser especialmente destacado en las obras Postscriptum no científico y definitivo a Migajas filosóficas, donde el seudónimo Climacus jocosamente de cuestiona si el cristianismo puede ser una cuestión de geografía, y también en la polémica final de El instante, donde Kierkegaard explícitamente declara que el cristianismo del Nuevo Testamento está muerto y, de ese modo, parece sintetizar las 95 tesis de Lutero en apenas una. Ya el tema de la revelación divina es igualmente perceptible en innumerables de sus escritos y destacaríamos aquí dos de ellos que eran del conocimiento del teólogo suizo: El libro sobre Adler y Sobre la diferencia entre un genio y un apóstol. Ambos tratan del tema de la revelación y de la afirmación de la autoridad apostólica. En el primero, a través de un caso real ocurrido al pastor Adler, que afirmaba haber recibido una revelación divina, que lo incitaba a abandonar su oficio de profesor de Lógica y de pastor de la Iglesia luterana, Kierkegaard observa en tal episodio una sátira tanto de la Iglesia como de la especulación hegeliana, ambas marcadas por la confusión entre cultura y cristianismo de su época. Ya en el segundo texto, que como el primero pertenece a los escritos póstumos del autor danés, Kierkegaard apunta que el telos de un apóstol está en la trascendencia (como en el caso de Paulo, por ejemplo), como el telos de un genio, tal como Platón o Shakespeare, reside en la inmanencia. Las dos

${ }^{4}$ Barth, K. A Carta aos Romanos, ed. por Cornelis van der Kooi e Katja Tolstaja, trad. de Uwe Wegner, São Leopoldo: Sinodal, 2016, p. 7. 
esferas no deben mezclarse y la autoridad de un apóstol no viene, por lo tanto, de su brillo como genio, sino de lo trascendente que lo inspira y legitima en su misión. Como reconoce el propio Barth, ya el primer capítulo de la Carta a los romanos, citando directamente a Kierkegaard, "la vocación apostólica es un hecho paradójico"s.

Cabe resaltar que Barth, como bien observan Cornelis van der Kooi y Katja Tolstaja, hizo el primer esbozo de la obra en 1919, pero luego la rehízo en 1922 (versión oficialmente reconocida) en virtud de haber recibido diversas influencias en ese periodo de lecturas e interpretaciones: "Barth enumera una serie de factores que lo llevaron a efectuar una revisión fundamental: el estudio progresivo de Paulo; la lectura de Overbeck; una mejor inserción en el mundo de las ideas de Platón y Kant, gracias a su hermano Heirich Barth y la influencia de Kierkegaard y Dostoievski”"

El teólogo suizo disponía y leía al pensador danés en las ediciones alemanas de Gottsched, Pfleiderer y Schrempf (1909-1922, 12 volúmenes), y poseía ediciones alemanas de la época de Migajas filosóficas, Postscriptum no científico y definitivo a Migajas filosóficas, El libros del juez (parte de los Diarios), Ejercitación en el cristianismo, Las obras del amor?. De ese modo, cabe apuntar también algunos de los límites de su lectura y abordaje, incluso en virtud de las ediciones que poseía y considerando que en aquel momento todavía no se disponía de ediciones más críticas de la obra kierkegaardiana en alemán, lo que ciertamente no impidió al teólogo Barth hacer un excelente uso de los recursos que tenía disponibles. En ese sentido, la alerta de Barrett nos parece fundamental: "Por causa de su centralidad en la primera apropiación de Barth sobre Kierkegaard, las citas de Kierkegaard en la Carta a los romanos deben ser exploradas con algún cuidado"8.

En su prefacio a la segunda edición, por ejemplo, Barth apunta hacia la diferencia cualitativa interminable entre tiempo y eternidad, así como la diferencia absoluta entre Dios y el hombre, tal como afirma Schwöbel: "Gran parte de la retórica expresionista de Romanos pretende enfatizar

${ }^{5}$ Barth, A Carta aos Romanos, p. 71.

${ }^{6}$ Barth, A Carta aos Romanos, p. 8.

${ }^{7}$ Mayores detalles sobre las ediciones de las cuales Karl Barth disponía pueden ser observados en las pp. 35 y 36 de la edición brasileña de la obra, esto es, en la lista de las abreviaturas. Informaciones aún más precisas pueden ser encontradas en el artículo de Lee Barret, igualmente citado en nuestra bibliografía final.

${ }^{8}$ Barrett, L. "Karl Barth: The Dialectic of Attraction and Repulse" en Kierkegaard's influence on Theology - Tome I: German Protestant Theology, ed. por Jon Stewart, Burlington: Ashgate, 2012, p. 26. 
la distinción absoluta entre Dios y el mundo. El famoso apelo de Barth a Kierkegaard en el prefacio de la segunda edición". El mismo señalamiento es hecho por Barrett: "La influencia de Kierkegaard es más evidente en la segunda edición de la Carta a los romanos de Barth, tanto en los textos citados como en los temas articulados. En este texto, Barth repite la frase de Kierkegaard, 'la distinción cualitativa'" 10.

Percibimos aquí con gran claridad una lectura atenta de los temas motivadores de la reflexión kierkegaardiana en obras como El concepto de la angustia y Migajas filosóficas. Ya en su primer capítulo de la Carta a los romanos, surge claramente la palabra paradoja, tomada aquí no sólo en su sentido amplio tanto en el campo de la historia de la teología como en las discusiones lógicas, sino directamente de la reflexión kierkegaardiana al ser, incluso, citado el nombre del autor danés entre paréntesis después de su uso. En ese mismo capítulo, el pensador suizo demuestra también conocer una importante obra de Kierkegaard: Ejercitación en el cristianismo. Ahí, a través de la reflexión acerca de la temática Dios-hombre, el teólogo recupera un tema crucial de la tradición teológica que es la encarnación divina y lo aplica con un lenguaje muy kierkegaardiano, a saber, lo incógnito y lo desconocido, el Dios que no se captura sólo por un dado tipo de razón, es el Dios que invita a los hombres a vivir una vida diferente.

Esa misma reflexión presente en la lectura que Barth hace de Ejercitación en el cristianismo es retomada en el tercer capítulo para enfatizar en dos aspectos singulares del cristianismo. El primero es que este se constituye en un escándalo, esto es, en una quiebra de la ley y de aquello que era esperado por una religión tradicional. El segundo es que no puede ser comunicado de manera directa, puesto que no se trata de un conocimiento objetivo o de la esfera científica. De ese modo, el cristianismo barthiano se afirma aquí como muy paulino y kierkegaardiano. En efecto, supera tanto el escándalo -puesto que supera la ley- que es una especie de locura- ya que ofende la mentalidad lógica de los griegos.

Curiosamente, en ese mismo capítulo, inspirado también por una reflexión de El concepto de la angustia, Barth toca un tema tan cercano a Kierkegaard, dejándolo absolutamente explícito en una nota a pie de página: el tema del instante, donde el cristianismo se afirma en el tiempo y donde la eternidad y el tiempo se tocan, formando el instante.

9 Schwöbel, C. “Theology” en The Cambridge Companion to Karl Barth, ed. por J. Webster, Cambridge: CUP, 2000, p. 20.

${ }^{10}$ Barrett, Karl Barth: The Dialectic of Attraction and Repulse, p. 8. 
En el cuarto capítulo, Barth toca una obra kierkegaardiana que parece fundamental para todo teólogo, a saber, La enfermedad mortal. Aquí es recuperado otro tema kierkegaardiano importante a través de una reflexión en la base del cristianismo: el tema del individuo, tan apreciado por la tradición cristiana desde los primordios y trabajado magistralmente en el tema de la conciencia en La enfermedad mortal. Siguiendo esta secuencia del texto paulino, el pensador se depara con la paradigmática figura de Abraham, pero de forma muy provocativa, aunque la reflexión kierkegaardiana esté aquí claramente presente, no hay mención explícita, al menos en ese momento, a la obra Temor y temblor, donde la figura del patriarca es central. Una hipótesis para eso es que, tal vez, el pensador suizo haya optado por una exégesis más enfática del texto bíblico y no se haya interesado, al menos en ese momento, por una discusión psicológica desenvuelta en Temor y temblor.

Sin embargo, en ese mismo capítulo, Barth muestra un conocimiento no sólo de los textos kierkegaardianos, sino del contexto donde este se insertó. Llega a citar hasta a Martensen, célebre profesor danés y obispo de Copenhague en el momento en que Kierkegaard critica fuertemente a la Iglesia y a la cultura en El instante. El teólogo llega a decir, en una nota al pie, que el sermón fúnebre de elogio al antiguo obispo Mynster, proferido por Martensen, donde el fallecido obispo es tomado como un "testigo de la verdad en la carrera de los apóstoles” será el catalizador para la crítica kierkegaardiana a ser desarrollada en El instante pues, a los ojos del danés, sería necesario aclarar lo que efectivamente se dice cuando nombramos a alguien como un "testigo de la verdad en la carrera de los apóstoles".

$\mathrm{Al}$ disertar sobre el nuevo ser humano propuesto en la epístola a los romanos, Barth utiliza nuevamente a Kierkegaard, esta vez haciendo uso de su Postscriptum no científico y definitivo a Migajas filosóficas, donde el acto de creer es tomado como una especie de pasión infinita, proporcionando así la tonalidad de la reflexión que desarrollará en el quinto capítulo de su la Carta a los romanos.

Al tratar el tema de la libertad y del límite de la religión en el séptimo capítulo, Barth muestra nuevamente que está familiarizado con detalles de la interpretación kierkegaardiana y de un amplio contexto cultural. Aquí, en una nota al pie, apunta que la pieza Brand del noruego Ibsen habría estado abiertamente inspirada en Kierkegaard y que su personaje principal es, en el fondo, una figura kierkegaardiana en contradicción con sus dilemas ético- morales. En ese mismo capítulo, el pensador diserta acerca del “coraje 
dialéctico de Kierkegaard"11, lo que demuestra una nueva familiaridad, por ejemplo, con el tema desarrollado por el pensador danés acerca de la figura del pensador dialéctico. Por eso, igualmente no parece casual que la tradición barthiana también será tomada por algunos como "teología dialéctica", lo que parece muy significativo.

Mientras, en el octavo capítulo aparece una mención curiosa. Barth habla acerca del "venenoso superpietismo de Kierkegaard" ${ }^{2}$. Tal crítica merece ser vista de modo cuidadoso y en sentido estricto, precisa ser investigada dentro del contexto del luteranismo danés. El pietismo no deja de ser una especie de romanticismo aplicado al luteranismo. Después del establecimiento del protestantismo oficial, el luteranismo también vivió su periodo escolástico, esto es, una especie de escolástica protestante. De ese modo, el pietismo buscaba un tipo de experiencia íntima y de piedad individual, yendo más allá de una explicación cristiana. No obstante, un "superpietismo", como el que Barth le atribuyó a Kierkegaard, puede constituirse en una enfermedad y no ya en medicina, o sea, en una dosis que no es beneficiosa, sino que, al contrario, puede envenenar a alguien justamente por ser desmedida.

En ese mismo capítulo hay otra mención que merece ser destacada: aquella donde Barth se refiere a Spinoza y comprende cómo este pensador fue importante en la construcción kierkegaardiana. Cita literalmente la expresión de Spinoza utilizada en Ética, a saber: sub specie aeterni ${ }^{13}$. A sus ojos toda la discusión paulina que toca en el tema de que el cristiano debe ser guiado por el Espíritu Santo implica verdaderamente un poco de aprender a mirar el mundo bajo la perspectiva de la eternidad, hecho mostrado por Spinoza y recuperado por Kierkegaard.

En la parte titulada por Barth de capítulos 12-15, aparece una mención extremadamente interesante sobre la idea del prójimo en Kierkegaard, lo que ciertamente interesa desde el punto de vista teológico, pero también en la discusión ética. Aquí el teólogo suizo cita directamente la obra principal del pensador danés en ese aspecto, a saber, Las obras del amor. A diferencia del modelo griego de amor de predilección, Barth se preocupa por el tema paulino de la multiplicidad del grupo de cristianos, esto es, de la variedad de personas que lo componían. El objetivo del teólogo es recuperar el tema del prójimo como aquel que coloca a todos los hombres en iguales condiciones

\footnotetext{
${ }^{11}$ Barth, A Carta aos Romanos, p. 267.

12 Ibíd., p. 289.

${ }^{13}$ Ibíd., p. 314.
} 
ante Dios. En otras palabras, somos todos nosotros prójimos de aquellos que nos rodean y esa igualdad está garantizada por el amor común de Dios a todos y por el imperativo "debes amar", sin hacer ninguna distinción personal por ningún motivo. El amor de ese Dios es, por lo tanto, nuestra garantía y debe ser nuestra práctica.

Concluida esta etapa, donde buscamos separar pequeñas porciones de la influencia kierkegaardiana en Karl Barth, me parece que ahora estamos preparados para observar a Barth dentro del contexto del existencialismo europeo del siglo XX.

\section{La Carta a los romanos en el contexto del existencialismo del siglo XX}

El momento de producción de la Carta a los romanos de Karl Barth es un tiempo de crisis, después del contexto de la Primera Guerra Mundial. El filósofo italiano Luigi Pareyson, en su clásica obra Studi sull'esistenzialismo, apunta cuánto la obra del teólogo suizo y la de Karl Jaspers son vitales dentro de tal contexto y cuánto ambas obras, cada una, a su modo, son producidas bajo el signo de la filosofía kierkegaardiana:

En 1919 sale Römerbrief de Karl Barth y Psychologie der Weltanschauungen de Karl Jaspers. Dos autores muy diversos: de una parte, el teólogo Barth, con su comentario a la carta a los Romanos de San Pablo, coloca las bases de la teología dialéctica o teología de la crisis, que tanta influencia ejerció en el mundo protestante alemán, pero también en el francés. De otra parte, Jaspers, que de la psiquiatría se volvió psicólogo... Mas una extensa sección del libro de Jaspers está reservada a Kierkegaard, y toda la teología de Barth está íntimamente permeada por el pensamiento del agudo y atormentado teólogo danés de la primera mitad del siglo pasado. Ese interés común por el pensamiento kierkegaardiano es el que conecta más íntimamente de lo que parece a primera vista, las dos obras citadas. En esa primera etapa de la historia del existencialismo se entiende la importancia para cada concepción existencialista de la filosofía kierkegaardiana. La especulación kierkegaardiana se reproduce, sobre todo, sobre el concepto de existencia, que significa la exasperada singularidad del hombre desnudo ante Dios... El pensamiento kierkegaardiano, de hecho, es el emblema de la revalorización existencialista del singular ${ }^{14}$.

${ }^{14}$ Pareyson, Studi sull'esistenzialismo, p. 11. 
En otras palabras, Pareyson sitúa a Kierkegaard como el eslabón primordial de la historia del existencialismo del siglo XX. Su repercusión del tema del singular, tan cercano a él por su relación con la filosofía antigua como por el cristianismo, posee sin dudas un apelo también a la tradición protestante, al final el tema del individuo es por excelencia un tema también protestante. En ese sentido, la Carta a los romanos de Barth no deja de flirtear con eso al colocar al singular delante de Dios como uno de los problemas existenciales de su teología.

Hay un pasaje singular de Hannah Arendt en un texto titulado Søren Kierkegaard, que nos parece ejemplar para demostrar cabalmente tal tesis. Afirma la pensadora que, en los tiempos modernos, donde la secularización y la desconfianza se instalan, no es necesario sólo afirmar al cristianismo, sino que es necesario afirmarlo cuando todos lo niegan a través de tesis y argumentos muy bien elaborados. Por eso, "ser radicalmente religioso en tal mundo significa estar solo no solamente en el sentido de que la persona se postra ante Dios, sino también en el sentido de que nadie más se postra ante Dios" ${ }^{15}$. En otras palabras, no parece haber comunidad cristiana, sino algunos individuos que todavía buscan mantener la integralidad del mensaje cristiano. Se trata, por lo tanto, de una lucha existencial, de aquello que Miguel de Unamuno denominó La agonía del cristianismo ${ }^{16}$.

También el filósofo italiano Giacomo Marramao señala con sutileza aquello que denomina como Kierkegaard Renaissance, esto es, el influjo de la obra kierkegaardiana y de sus temas como centrales para toda una filosofía que se hará en la Europa de ese periodo. Tal filosofía también coloca en jaque un debate acerca del tema de la secularización, por eso además del ya citado Jaspers, surge al lado de este el autor de Christentum und Kultur, el célebre Overbeck, amigo personal de Nietzsche y pensador al que influenciará directamente la Carta a los romanos de Karl Barth:

Sin embargo, no menos significativa -en términos de la evolución del concepto de secularización - es la circunstancia de que la fecha de publicación póstuma de Christentum und Kultur (1919) coincida justamente con el momento de "Kierkegaard Renaissance", representada por obras como Psychologie der Weltanschaunngen [Psicología de las concepciones de mundo] de Karl Jaspers y el Römerbrief [Epístola a los romanos] de Karl Barth: una 'coincidencia' que ya por sí sólo pone la cuestión de la correlación temática entre teología
${ }^{15}$ Arendt, Compreender - formação, exílio e totalitarismo, p. 76.
${ }^{16}$ Unamuno, M. A Agonia do cristianismo, trad. de Arthur Guerra, Lisboa: Cotovia, 1991. 
negativa e incubación teológica ochocentista de la 'rebelión a Hegel'. De hecho, es en el interior de esa incubación que se manifestará la cuestión del individuo como paradoja de una existencia 'deshumanizada' en la época de la completa mundanización (Verweltlichung): la fe, entendida en el sentido de Kierkegaard, es aquella 'paradoja' por la cual lo singular es superior a lo general $^{17}$.

Entonces, Arendt parece tener razón cuando dice que "Kierkegaard fue el primer pensador que vivió en un mundo semejante al nuestro, esto es, un mundo totalmente secularizado oriundo del Iluminismo"18. Así, es bastante significativo pensar que Karl Barth, lector de Kierkegaard, se inserta exactamente en ese contexto y, a su modo, intenta articular respuestas para algunas preguntas que ya existían en el tiempo del autor danés. En otras palabras, ¿cómo la teología dialéctica del siglo XX sobrevivirá al desafío de la secularización y conseguirá articular un discurso válido para los hombres de su tiempo y será fiel al mandamiento divino? Semejante tema debe ser la preocupación de un teólogo.

Cabe destacar que tal intercambio entre teólogos y filósofos nunca fue ajeno al mundo germánico, aunque, tal vez, esto necesite de una mejor explicación en otros contextos, donde hay una separación más radical entre filosofía y teología, así como entre Estado y religión. Al mismo tiempo, eso no quiere decir que filosofía y teología sean lo mismo, pero como apunta Leo Strauss en su texto La influencia mutua entre teología y filosofía, sólo significa que el filósofo puede estar abierto a los desafíos de la teología y el teólogo puede estar abierto a los desafíos de la filosofía:

Cuando intentamos retornar a las raíces de la civilización occidental, enseguida observamos que la civilización occidental tiene dos raíces que están en conflicto una con la otra, la bíblica y la filosofía griega, y eso para comenzar con una observación de veras desconcertante. Aun así, esa comprensión también tiene algo reconfortante y tranquilizante. La vida misma de la civilización occidental es la vida entre dos códigos, una tensión fundamental. Por eso, no hay ninguna razón intrínseca a la propia civilización occidental, en su constitución fundamental, por la cual esta debe abdicar de la vida. Pero ese pensamiento tranquilizador sólo se justifica si vivimos esa vida, esto es, si vivimos ese conflicto. Nadie puede ser al mismo tiempo filósofo y teólogo, ni, de hecho, una tercera cosa que esté más allá del conflicto entre filosofía y

${ }^{17}$ Marramao, G. Céu e terra - genealogia da secularização, trad. de Guilherme Alberto Gomez de Andrade, São Paulo: Unesp, 1997, p. 43.

${ }^{18}$ Arendt, Compreender-formação, exílio e totalitarismo, p. 75. 
teología, o una síntesis de ambos. Pero cada uno de nosotros puede y debe ser uno de los dos, el filósofo abierto al desafío de la teología o el teólogo abierto al desafío de la filosofía ${ }^{19}$.

En ese sentido, un excelente panorama del contexto intelectual de la época es igualmente proporcionado por Karl Löwith quien, en su obra Mi vida en Alemania antes y después de 1933, apunta que su generación estuvo profundamente influida por la lectura de dos obras capitales producidas en el periodo: La decadencia de Occidente, de Spengler (1918) y Carta a los romanos, de Karl Barth (1919). A pesar de colocarla un punto debajo de la obra de Spengler, Löwith elogia profundamente la obra del teólogo suizo:

Algo parecido, aunque su efecto fuese más reducido, significó la Carta a los romanos de Karl Barth que apareció en el mismo tiempo. También en esa obra abundaba la negación del progreso, llegando a conclusiones teológicas del colapso de la cultura. La desconfianza en las soluciones humanas fomentadas por la guerra, condujo a Barth del socialismo cristiano a una teología radical que negaba a partir de su raíz cualquier 'desarrollo' del cristianismo. Los escritos de Spengler y de Barth fueron los libros que despertaron en nosotros mayor entusiasmo en la época marcada por el fin de la Primera Guerra Mundial ${ }^{20}$.

Según Löwith, además de su tributo intelectual, Barth era merecedor de un reconocimiento político. Especialmente para él, que había sido orientado por Heidegger, la posición política del teólogo en el contexto europeo del periodo es algo que merece ser destacado. Por eso, Löwith apunta la diferencia crucial entre la postura del discurso de Heidegger aliado al nacismo y el discurso de Barth como su valiente oponente:

Un mes después de que Heidegger pronunciara su discurso, K. Barth escribió su manifiesto teológico (Theologische Existenz heute), en el cual abogaba contra la asimilación al poder del momento. Este escrito fue la única manifestación seria de una oposición intelectual a aquella época desgarradora y todavía permanece como tall ${ }^{21}$. p. 249.

${ }^{19}$ Strauss, Fé e Filosofia Política - a correspondência entre Leo Strauss e Eric Voegelin,

${ }^{20}$ Löwith, K. Mi vida en Alemania antes y después de 1933 - un testimonio, trad. de Ruth Zauner, Madrid: Visor, 1992, pp. 46-47.

${ }^{21}$ Ibíd., p. 57. 
Es decir, además de las posiciones políticas de Barth, tal vez fuera estimulante observar el aspecto político del pensamiento paulino, especialmente aquel que es expuesto en la epístola a los romanos, aplicado a un tipo de relectura contemporánea como la de Badiou que, por una perspectiva confesadamente ateísta, parece estar fascinado, en especial cuando piensa en el tema del amor, en cuánto la propuesta paulina se firma como revolucionaria en la medida en que esta implica una quiebra de la legalidad de la religión tradicional y coquetea con un individuo nuevo y universalizado. No obstante, Badiou es cauteloso, pues no piensa que el cristiano vive sin ley, sino en una especie de "existencia de una ley transliteral, de una ley del espíritu"22. Así, si Cristo es el fin de la ley (Rom 10,4) y el amor es la ejecución de la ley (Rom 13, 10), Badiou parece tener razón: "Bajo la condición de la fe, de la convicción declarada, el amor nombra una ley no literal, que da al sujeto fiel su consistencia y efectúa en el mundo la verdad pos acontecimiento" ${ }^{23}$. Ciertamente tal pista merece ser seguida con más detenimiento y detalladamente, lo que no será posible hacer aquí.

\section{Conclusiones}

El presente artículo se concentró, tanto en virtud de su delimitación como de la amplitud del propio tema, en tres aspectos esenciales. En la introducción, se pretendió, sin adentrar en ninguna minucia teológica o exegética, proveer un poco del contexto de producción de la epístola a los romanos y puntuar cuestiones que deben ser tenidas en cuenta en un análisis como el que emprendimos.

El segundo aspecto consistió en un análisis, también dentro de los límites posibles y de algunos recortes previamente delimitados, acerca de la influencia kierkegaardiana en Carta a los romanos de Karl Barth. Para eso, nos concentramos en señalar aspectos del pensamiento kierkegaardiano y su efectiva apropiación por el teólogo suizo. El objetivo fue colocar dos autores importantes de los siglos XIX y XX en diálogo claro y abierto, deseando comprender sus respectivos contextos, tesis y polémicas. Para eso, algunas obras y conceptos kierkegaardianos fueron relacionados especialmente a

22 Badiou, A. São Paulo, trad. de Wanda Nogueira Caldeira Brant, São Paulo: Boitempo, 2009, p. 101.

${ }^{23}$ Ibíd., p. 102. 
partir de citas de Kierkegaard en el propio texto de Barth. Al final, como bien observó Barrett:

Kierkegaard ciertamente influyó en el desarrollo teológico de Barth, particularmente durante el período inicial de Barth, informando la manera en que conceptuó cuestiones fundamentales y articuló grandes temas. No obstante, la extensión y la naturaleza exacta de esa influencia son una cuestión de algún debate. Los tipos básicos de evidencias relevantes para esta cuestión se dividen en tres categorías: los propios relatos de Barth sobre su lectura de las obras de Kierkegaard, las citas directas de Barth de Kierkegaard en su literatura y las reflexiones retrospectivas explícitas de Barth sobre la influencia de Kierkegaard sobre él. Los dos primeros tipos de evidencia ayudan a determinar qué partes de su corpus eran familiares para Barth, un asunto crítico para entender la interpretación que Barth hace de Kierkegaard. Antes de intentar entender el significado de las apropiaciones de Barth y críticas de Kierkegaard, primero debemos determinar lo que Barth realmente sabía sobre sus escritos y, por lo tanto, qué impresión de Kierkegaard Barth fue capaz de formular ${ }^{24}$.

Por fin, el tercer punto del artículo se constituyó en un análisis del contexto de surgimiento del llamado existencialismo europeo del siglo XX, especialmente aquel que se produjo en suelo alemán. Aquí, por medio de autores como Pareyson, Arendt, Löwth, Strauss y Marramao, se intentó percibir un cuadro significativo de tal movimiento y de su relación con el tema de la secularización. El objetivo principal fue señalar que la obra de Barth es inspiradora directa del existencialismo a partir de la así llamada teología dialéctica y que, al recuperar el tema del individuo, tan cercano a la tradición cristiana y protestante, el teólogo suizo se inserta junto a Kierkegaard y otros importantes autores del período en los ciernes de aquello que podemos tomar por existencialismo. Así, creemos haber cumplido un papel meramente aproximativo de grandes autores en un contexto muy curioso y que, ciertamente, merece profundización y estudios detallados.

${ }^{24}$ Barrett, "Karl Barth: The Dialectic of Attraction and Repulse”, p. 7. 


\section{Bibliografía}

Alexander, David e Pat, O mundo da Bíblia, trad. de Lailah de Noronha, São Paulo: Paulinas, 1985.

Arendt, H. Compreender - formação, exílio e totalitarismo, trad. de Denise Bottmann, São Paulo: Companhia das Letras, 2008.

Badiou, A. São Paulo, trad. de Wanda Nogueira Caldeira Brant, São Paulo: Boitempo, 2009.

Barrett, L. "Karl Barth: The Dialectic of Attraction and Repulse" en Kierkegaard's Influence on Theology - Tome I: German Protestant Theology, ed. por Jon Stewart, Burlington: Ashgate, 2012, pp. 01-41.

Barth, K. A Carta aos Romanos, ed. por Cornelis van der Kooi e Katja Tolstaja, trad. de Uwe Wegner, São Leopoldo: Sinodal, 2016.

A Bíblia - tradução ecumênica, São Paulo: Loyola/Paulus, 1995.

Jaeger, W. Cristianismo primitivo e paideia grega, trad. de Louro Perez, Lisboa: Edições 70, 2002.

Kierkegaard, S.A. Book on Adler, trad.de Edna y Howard Hong, New Jersey: Princeton University Press, 1998.

— O Conceito de angústia, trad. de Álvaro Valls, Petrópolis: Vozes, 2010.

— Ejercitación del cristianismo, trad. de Demetrio Gutiérrez Rivero, Madrid: Trotta, 2009.

_ La enfermedad mortal, trad. de Demetrio Gutiérrez Rivero, Madrid: Trotta, 2008 .

_ El Instante. Trad. de Andrés Roberto Albertensen, Madrid: Trotta, 2006.

- Kierkegaard's Journals and Notebooks vol. 1 - Journals AA-DD, trad. de Niels Jørgen Cappelørn, Alastair Hannay, David Kangas, Bruce H. Kirmmse, George Pattison, Vanessa Rumble y K. Brian Soderquist, New Jersey: Princeton University Press, 2007.

Kierkegaard's Journals and Notebooks vol. 2 - Journals EE-KK, trad. de Niels Jørgen Cappelørn, Alastair Hannay, David Kangas, Bruce H. Kirmmse, George Pattison, Vanessa Rumble e K. Brian Soderquist, New Jersey: Princeton University Press, 2007.

Kierkegaard's Journals and Notebooks vol. 3 - Journals 1-15 trad. de Niels Jørgen Cappelørn, Alastair Hannay, David Kangas, Bruce H. Kirmmse, George Pattison, Vanessa Rumble e K. Brian Soderquist, New Jersey: Princeton University Press, 2010. 
- Kierkegaard's Journals and Notebooks vol. 4 - Journals NB-NB5, trad. de Niels Jørgen Cappelørn, Alastair Hannay, David Kangas, Bruce H. Kirmmse, George Pattison, Vanessa Rumble e K. Brian Soderquist, New Jersey: Princeton University Press, 2011.

Kierkegaard's Journals and Notebooks vol. 5 - Journals NB6-NB10, trad. de Niels Jørgen Cappelørn, Alastair Hannay, David Kangas, Bruce H. Kirmmse, George Pattison, Vanessa Rumble e K. Brian Soderquist, New Jersey: Princeton University Press, 2012.

Kierkegaard's Journals and Notebooks vol. 6 - Journals NB11-NB14, trad. de Niels Jørgen Cappelørn, Alastair Hannay, David Kangas, Bruce H. Kirmmse, George Pattison, Vanessa Rumble e K. Brian Soderquist, New Jersey: Princeton University Press, 2013.

Kierkegaard's Journals and Notebooks vol. 7 - Journals NB15-NB20, trad. de Niels Jørgen Cappelørn, Alastair Hannay, David Kangas, Bruce H. Kirmmse, George Pattison, Vanessa Rumble e K. Brian Soderquist, New Jersey: Princeton University Press, 2014.

- Kierkegaard's Journals and Notebooks vol. 8 - Journals NB21-NB25, trad. de Niels Jørgen Cappelørn, Alastair Hannay, David Kangas, Bruce H. Kirmmse, George Pattison, Vanessa Rumble e K. Brian Soderquist, New Jersey: Princeton University Press, 2015.

- Kierkegaard's Journals and Notebooks vol. 9 - Journals NB26-NB30, trad. de Niels Jørgen Cappelørn, Alastair Hannay, David Kangas, Bruce H. Kirmmse, George Pattison, Vanessa Rumble e K. Brian Soderquist, New Jersey: Princeton University Press, 2017.

— Obras do amor, trad. de Álvaro Valls, Petrópolis: Vozes, 2005.

_ Ponto de vista explicativo da minha obra como escritor, trad. de João Gama, Lisboa: Edições 70, 1986.

Pós-Escrito às Migalhas Filosóficas v. I e II, trad. de Álvaro Valls, Petrópolis: Vozes, 2013-2016.

— Temor e Tremor, trad. de Elisabete Sousa, Lisboa: Relógio D’Água, 2009.

Leenhhardt, F.J. Epístola aos Romanos- comentário exegético, trad. de Waldyr Carvalho Luz, São Paulo: Aste, 1969.

Löwith, K. Mi vida en Alemania antes y después de 1933 - un testimonio, trad. de Ruth Zauner, Madrid: Visor, 1992.

Marramao, G. Céu e terra - genealogia da secularização, trad. de Guilherme Alberto Gomez de Andrade, São Paulo: Unesp, 1997.

Pareyson, L. Studi sull'esistenzialismo, Milano: Mursia, 2001. 
Schwöbel, C. "Theology” en The Cambridge Companion to Karl Barth, ed. por J. Webster, Cambridge: CUP, 2000, pp. 17-36.

Strauss, L; Voegelin, E. Fé e Filosofia Política - a correspondência entre Leo Strauss e Eric Voegelin, trad. de Pedro Sette-camara, São Paulo: Editora É, 2017.

Unamuno, M. A Agonia do cristianismo, trad. de Arthur Guerra, Lisboa: Cotovia, 1991. 